\title{
Le facteur de croissance nerveuse NGF : rôle dans la plasticité et la maintenance fonctionnelle de la cellule neuronale
}

Le NGF (nerve growth factor) est le premier connu d'une famille de facteurs nécessaires à la survie de populations cellulaires particulières. Il joue un rôle essentiel dans la survie et la différenciation terminale des neurones sensitifs et de ceux du système sympathique périphérique. Dans le système nerveux central, les neurones cholinergiques y sont également sensibles. Chez l'adulte, le NGF pourrait être nécessaire à la récupération anatomique et fonctionnelle de tissus nerveux lésés. Il pourrait aussi s'opposer aux phénomènes pathologiques de dégénérescence neuronale dans des affections comme la maladie d'Alzheimer et les polyneuropathies diabétiques.

\section{Philippe Brachet}

\section{ADRESSE ET TIRÉS À PART}

P. Brachet : directeur de recherche à l'Inserm, docteur ès sciences. Inserm U. 298. Centre hospitalier et universitaire, 49033 Angers Cedex, France. a greffe d'explants de tumeurs dans la cavité péritonéale d'embryon de poulet ne paraît pas être la meilleure façon d'obtenir des informations sur le développement du système nerveux.

C'est pourtant grâce à ce moyen peu conventionnel que la notion de facteur neurotrope a émergé. On en doit le principe expérimental à E. Bueker, qui procédait à de telles transplantations, à partir de sarcomes murins. Il avait constaté que certains greffons étaient colonisés par des terinaisons sensorielles, et que les ganglions hébergeant les corps cellulaires des neurones impliqués dans ce processus devenaient plus volumineux. R. Levi-Montalcini et V. Hamburger [1] ont repris ce système expérimental, et démontré que l'innervation sympathique répondait elle aussi à la présence des mêmes greffons, la stimulation de la croissance nerveuse allant jusqu'à provoquer une invasion des vaisseaux sanguins. Les neurones moteurs, en revanche, paraissaient insensibles à l'action trophique du transplant. Il existait donc une spécificité d'action de l'effet promoteur 
prodigué par le greffon tumoral sur le tissu nerveux. Un autre aspect important tenait au fait que le contact physique entre le transplant tumoral et les cellules nerveuses ou leurs prolongements était inutile : un greffon placé sur la membrane chorio-allantoïdienne provoquait à distance un développement anormal de l'innervation sympathique. Et surtout, la culture in vitro, dans un milieu semi-solide, d'un explant tumoral et d'un ganglion achevait de démontrer que l'effet neurotrophique était induit par une substance diffusible. Plus tard, S. Cohen, qui tentait de purifier le facteur neurotrophique, découvrait sa présence en quantité phénoménale dans le venin de certains serpents et la glande sousmaxillaire de souris mâle. La purification du nerve growth factor (NGF), à partir de ces sources, a permis l'obtention d'anticorps. L'injection de ceux-ci durant la vie périnatale entraîne une destruction massive de l'innervation sympathique, ce qui indique que du NGF endogène est effectivement produit au cours du développement, et que ce facteur exerce bel et bien un effet trophique sur l'innervation sensible.

Depuis, des activités neurotrophiques variées ont été observées, dans des surnageants de diverses cultures de cellules ou des extraits d'organes. Elles agissent sur certaines populations de neurones sensoriels, sympathiques, parasympathiques, moteurs, ou encore sur des populations neuronales issues de régions variées du système nerveux central ; ces activités opèrent, en outre, à des temps variables du développement embryonnaire ou post-natal. Si l'on se doute, par conséquent, que le NGF n'est qu'un facteur neurotrophique parmi beaucoup d'autres, rares sont ceux qui ont été caractérisés au niveau moléculaire (figure 1). Ces facteurs constitueraient autant de messagers directement impliqués dans le dynamisme neurogénétique comme dans la stabilisation des connexions neuronales. Leur sécrétion par certains types cellulaires assurerait la survie des populations neuronales sensibles, leur différenciation termi-

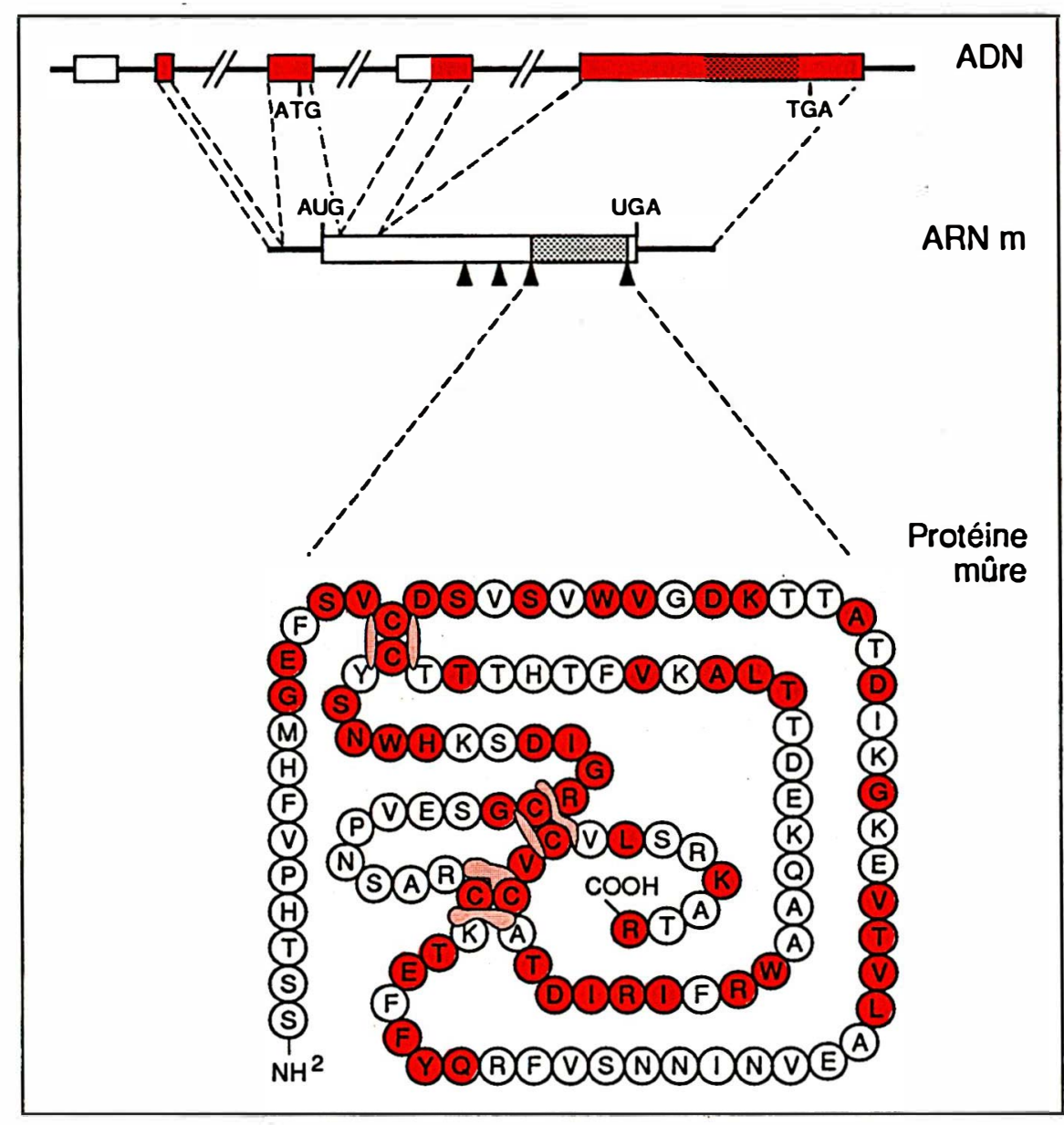

Figure 1. (1) Structure du gène NGF de souris montrant les différents exons marqués en trait épais. Ceux qui sont utilisés pour constituer I'ARNm le plus abondant trouvé dans la glande sous-maxillaire de souris mâle sont colorés en rouge. En pointillé, la région spécifiant le monomère du NGF mûr ld'après [2]). (2) Structure de I'ARNm du NGF correspondant, avec les régions 3' et $5^{\prime}$ non codantes, en trait fin, et la région codante (proNGF) en trait épais. Les symboles $\Delta$ sont situés au niveau de paires d'acides aminés basiques qui constituent des signaux probables de clivage du précurseur par des enzymes protéolytiques participant à la maturation du NGF. (3) Structure secondaire de la protéine NGF montrant les 3 ponts disulfures établis entre les six résidus cystéine soulignés en rose. Les acides aminés sont marqués en code à une lettre. Ceux imprimés en rouge, qui incluent les six résidus cystéine, sont rigoureusement conservés dans deux autres protéines qui présentent donc une structure primaire et secondaire voisine de celle du NGF : le brain-derived neurotrophic factor ou BDNF - un facteur neurotrophique actif sur les neurones sensoriels et certains neurones centraux [3] - et la neurotrophine 3, récemment clonée sur la base de cette analogie structurale [44]. En revanche, un autre facteur de structure connue, le ciliary neurotrophic factor ou CNTF, qui est notamment actif sur des neurones des ganglions ciliaires, est sans analogie biochimique avec le NGF [4]. Par ailleurs, un facteur authentiquement mitogène comme le fibroblast growth factor basique, ou FGFb, exerce des activités neurotrophiques typiques sur certains neurones centraux ou sur des lignées de cellules neuronales [5]. Les insulin-like growth factors (IGF) et peut-être certaines lymphokines pourraient également avoir des actions neurotrophiques. 


\section{RÉFÉRENCES}

1. Levi-Montalcini $\mathrm{R}$, Hamburger $\mathrm{V}$. Selective growth stimulating effects of mouse sarcoma on the sensory and sympathetic nervous system of the chick embryo. $J$ Exp Zool 1951; 116 : 321-62.

2. Selby MJ, Edwards R, Sharp F, Rutter WJ. Mouse nerve growth factor gene : structure and expression. Mol Cell Biol $1987 ; 7: 3057-64$.

3. Leibrock J, Lottspeich F, Hohn A, et al. Molecular cloning and expression of brainderived neurotrophic factor. Nature 1989 ; 341 : 149-52.

4. Lin HLF, Mismer DJ, Armes LG, Butler III ET, Vannice JL, Collins F. Purification, cloning and expression of ciliary neurotrophic factor (CNTF). Science 1989; 246: 1023-5.

5. Anderson KJ, Dam D, Lee S, Cotman CW. Basic fibroblast growth factor prevents death of lesioned cholinergic neurons in vivo. Nature 1988 ; 332 : 360-1.

6 . Shelton DL, Reichardt LF. Expression of the beta nerve growth factor gene correlates with the density of sympathetic innervation in effector organs. Proc Natl Acad Sci USA 1984; 81 : 7951-5.

7. Korsching S, Auburger G, Heumann R, Scott $J$, Thoenen $H$. Levels of nerve growth factor and its mRNA in the central nervous system of the rat correlate with cholinergic innervation. EMBO J 1985; 4 : 1889-93. 8. Stach RW, Perez-Polo JR. Binding of nerve growth factor to its receptor. $J$ Neurosci Res $1987 ; 17: 1-10$.

9. Greenberg ME, Greene LA, Ziff EB Nerve growth factor and epidermal growth factor induce rapid transient changes in proto-oncogene transcription in PC12 cells. $J$ Biol Chem 1985 ; 260 : 14101-10.

10. Heumann R, Schwab M, Thoenen $H$. A second messenger required for nerve growth factor biological activity? Nature $1981 ; 292: 838-40$

11. Johnson EM, Taniuchi M, Di Stefano PS. Expression and possible function of nerve growth factor (NGF) receptors on Schwann cells. Trends Neurosci 1988 ; 11 . 299-304.

12. Heumann R, Korsching S, Bandtlow $C$, Thoenen $H$. Changes of nerve growth factor synthesis in non neuronal cells in response to sciatic nerve transection. J Cell Biol 1987 ; 104 : 1623-31.

13. Rych KM, Luszynski JR, Osborne PA, Yip HK, Johnson EM. Nerve growth factor protects adult sensory neurons from cell death and atrophy caused by axotomy. $J$ Neurocytol 1987 ; 16 : 261-7.

14. Martin DP, Schmidt RE, Di Stefano P, Lowry OH, Carter JG, Johnson EM. Inhibitors of protein synthesis and RNA synthesis prevent neuronal cell death caused by nerve growth factor deprivation. $J$ Cell Biol 1988; 106 : 829-44.

15. Weber M, Raynaud B, Delteil C. Molecular properties of a cholinergic differentiation factor from muscle-conditioned medium. I Neurochem 1985 ; 45 : 1541-7.

16. Saadat S, Sendtmer M, Rohrer H. Ciliary neurotrophic factor induces cholinergic differentiation of rat sympathetic neurons in culture. J Cell Biol 1989; 108 : nale, l'élaboration, puis le maintien d'une innervation appropriée. $\mathrm{La}$ spécificité d'action de ces facteurs serait conférée par la présence sur les cellules nerveuses de récepteurs caractéristiques pour chaque facteur neurotrophique. Les recherches effectuées sur le NGF confortent au moins pour partie cette vision.

\section{Un peu de biochimie}

Chez les mammifères, on trouve du NGF en quantité abondante dans de rares glandes exocrines, toutes liées. au sexe mâle : glande sous-maxillaire de souris mâle (sujette à dimorphisme sexuel dans cette espèce), prostate de cochon d'Inde ou vésicules séminales de taureau. Le rôle biologique du facteur sécrété dans la salive ou le liquide spermatique reste incompris. Cette situation a toutefois permis la purification du NGF, et donc sa caractérisation biochimique. Le NGF est un homodimère de masse molaire $26.500 \mathrm{Da}$, de point isoélectrique élevé $(9,3)$. Chaque monomère dérive d'un précurseur par clivage protéolytique. Les ADNc correspondant aux ARNm trouvés dans la glande sous-maxillaire de souris mâle ont été clonés, et la structure des précurseurs du NGF déduite des séquences nucléotidiques. Il existe chez la souris plusieurs ARNm qui diffèrent à leur extrémité 5 'terminale et sont susceptibles d'engendrer deux formes de proNGF présentant des différences de séquences à leur extrémité aminoterminale [2]. La séquence de 118 acides aminés qui constitue le NGF mûr est située à l'extrémité carboxyterminale de ces précurseurs (figure 1). Le NGF mûr, encore appelé BNGF, peut encore subir des clivages protéolytiques engendrant une forme légèrement plus courte, appelée NGF 2,5S. A noter qu'un octapeptide aminoterminal, clivé lors de la formation de NGF 2,5S, paraît doué de propriétés hyperalgésiantes. On ignore actuellement si les peptides produits lors de la maturation du proNGF exercent un rôle biologique.

Les teneurs en NGF des tissus périphériques ou de diverses parties du cerveau sont variables, mais faibles (de 0,1 à $10 \mathrm{ng} / \mathrm{g}$ de tissu). Un fait remarquable est que le contenu en
ARNm NGF de chaque tissu périphérique est étroitement corrélé à la densité de l'innervation sympathique (ou aux teneurs en catécholamines), un fait qui s'accorde bien avec le rôle prêté au facteur [6]. Dans le cerveau, les neurones catécholaminergiques, par exemple de la substance noire, paraissent insensibles au facteur. On sait, en revanche, que les neurones cholinergiques dont les corps cellulaires sont situés dans des structures basales du cerveau antérieur (septum, bande diagonale de Broca et noyau de Meynert) répondent au NGF. Ces neurones émettent des projections ascendantes vers le cortex cérébral et l'hippocampe, et c'est précisément dans ces deux structures que l'ARNm NGF est le plus abondant [7]. Cependant, on trouve des ARNm NGF et de l'immunoréactivité de type proNGF dans de nombreuses autres aires cérébrales, ce qui suggère que bien d'autres populations de neurones centraux sont sensibles au facteur.

La quantité de protéine NGF peut être mesurée à l'aide de dosages immunoenzymatiques à deux sites. L'emploi de cette méthode délicate, qui paraît raisonnablement à l'abri de pièges, a montré que les structures périphériques ou centrales les plus riches en ARNm NGF étaient également les plus riches en facteur protéique. Mais du NGF est aussi trouvé au niveau des corps cellulaires des neurones sensibles, comme les ganglions sympathiques dans le système nerveux périphérique ou le septum dans le cerveau, qui constituent des structures où l'ARNm NGF est fort rare. La présence de NGF s'explique par le fait qu'il a été capté par les terminaisons nerveuses au niveau de ses sites de production, puis transporté vers les corps cellulaires par un mécanisme de flux axonal rétrograde.

\section{Les récepteurs, pièces incontournables de la réponse}

Des récepteurs au NGF ont été trouvés sur la surface des neurones sensibles. Ils se répartissent en deux classes, selon leur affinité [8]. C'est ainsi que les récepteurs à forte affinité des neurones sympathiques ont 
une $\mathrm{Kd}$ égale à $10^{-11} \mathrm{M}$ alors que les récepteurs à faible affinité ont une $\mathrm{Kd}$ égale à $10^{-9} \mathrm{M}$. On dispose de fragments génomiques spécifiant ce dernier type de récepteur, ainsi que d'anticorps monoclonaux. Mais on ignore encore si le récepteur à forte affinité constitue une espèce moléculaire totalement distincte ou s'il est constitué, au moins pour partie, de la chaîne protéique formant le récepteur à faible affinité : la description de phénomènes pouvant s'interpréter comme une interconversion justifie cette dernière hypothèse. Ce sont clairement les récepteurs à forte affinité qui transduisent le signal neurotrope. L'interaction ligand-récepteur est suivie par des cascades de réactions secondaires mal connues, qui mettent notamment en jeu des protéines kinases de divers types. Mais la nature du messager secondaire directement engendré par le récepteur lors de son association au NGF reste inconnue. Les effets du facteur sont multiples et s'échelonnent sur des périodes de temps qui vont de la minute (extrusion de $\mathrm{Na}^{+}$par des neurones sympathiques en culture, accumulation temporaire de diacylglycérol et de phospho-inositol trisphosphate) à plusieurs heures ou jours (poussée neuritique avec tout un cortège de modifications métaboliques). Un processus rapide d'activation de proto-oncogènes ( $c$-fos, puis $c-m y c)$ [9] est associé à certaines de ces réponses. Notons enfin que la micro-injection de NGF dans des cellules sensibles n'est suivie d'aucun effet, ce qui suggère l'importance du récepteur dans le déclenchement des réponses neurotrophiques [10].

Quant aux récepteurs à faible affinité, ils peuvent apparaître sur certaines cellules non neuronales : les cellules de Schwann, par exemple, positionnent ce récepteur en culture in vitro. Après transection du nerf sciatique, les cellules de Schwann situées dans la partie distale synthétisent également le récepteur à faible affinité. Il a été proposé par $\mathrm{E}$. Johnson [11] que ce processus serait destiné à favoriser la régénération nerveuse. En effet, ces mêmes cellules deviennent également capables de sécréter du NGF [12]. Le facteur produit serait piégé par les récepteurs à faible affinité de façon à $m / s n^{\circ}$ y. ooi. 6 , novembre 90 limiter sa diffusion et permetre sa présentation au récepteur à forte affinité présent sur la fibre nerveuse en régénération. On peut raisonnablement supposer que la cellule de Schwann est susceptible de synthétiser d'autres facteurs neurotrophiques et que grâce à un mécanisme comparable, c'est l'ensemble des axones constitutifs du nerf dont la régénération est stimulée, chaque cellule nerveuse trouvant les apports trophiques appropriés.

\section{Mort, survie et différenciation terminale des neurones}

Les facteurs neurotrophiques peuvent être plus ou moins indispensables au cours du développement, pour ne devenir, peut-être, chez l'adulte que des molécules de confort, en dehors d'un rôle plus critique lors de processus de régénération nerveux. Les exemples de ce phénomène sont variés :

In vitro, la survie de nombreux neurones sensoriels isolés à partir d'embryons de poulet âgés d'une à deux semaines est très dépendante de la présence de NGF, mais cette exigence s'atténue et, au cours de la troisième semaine, d'autres facteurs - comme le BDNF, brain derived neurotrophic factor - prennent le relais. De même, si la survie in vitro des neurones sympathiques du ganglion supérieur cervical du rat nouveau-né nécessite elle aussi la présence de NGF dans le milieu de culture, les neurones issus de l'animal adulte semblent peu ou pas sensibles au facteur in vitro. In vivo, l'injection d'anticorps anti-NGF provoque une privation en facteur endogène. Durant la vie fotale, cette situation s'accompagne d'effets patents sur l'innervation sensorielle et sympathique, en diminuant les contenus en neurotransmetteurs voire en entraînant la mort de neurones. Lors de la vie post-natale, cet effet est limité à l'innervation sympathique, et il devient difficile à mettre en évidence chez l'animal adulte. Cela n'interdit pas que les processus de régénération axonale ou de bourgeonnement, consécutifs à des traumatismes, ne se réalisent que sous l'impulsion d'activités neurotrophiques. En effet, après axotomie locale, le bourgeonnement nécessaire à la réinnervation sensorielle de la peau de rat ne se produit que très partiellement chez des animaux immunisés contre le NGF, ou traités avec des anticorps anti-NGF. De façon plus frappante, on constate que l'atrophie ou la mort de neurones sensoriels, consécutive à leur axotomie, est empêchée (ou retardée) par un traitement local du nerf sectionné par du NGF [13].

Le fait que les neurones sympathiques du ganglion supérieur cervical du rat nouveau-né survivent in vitro, à condition qu'ils soient incubés en présence de NGF, a mené à la proposition que le phénomène de mort cellulaire, qui affecte normalement de façon massive ces neurones in vivo au cours de la première semaine de la vie post-natale, pourrait résulter d'une limitation en apport trophique. Seuls les neurones qui ont établi des connections appropriées, dans une atmosphère de compétition sans merci, recevraient le support indispensable en NGF et survivraient. Cette vision a peut-être un fond de vérité, mais il demeure que la mort neuronale reste importante lorsque le territoire d'innervation est accru, par exemple, par la greffe d'un membre. Réciproquement, un traitement par du NGF n'empêche que partiellement la mort des neurones dont le territoire d'innervation a été amputé. Il est donc vraisemblable que la mort de ces cellules dépend d'une programmation dont la limitation en facteur neurotrophique ne constitue qu'un élément. Le fait que, in vitro, la présence d'inhibiteurs métaboliques de la transcription ou de la traduction retarde la mort neuronale induite par la privation de NGF s'accorde avec cette notion, puisque ce phénomène suggère que la mort nécessite une expression génétique particulière [14].

Le rôle plastique des facteurs neurotrophiques persiste ensuite pour ce qui est de la fraction de neurones appelés à survivre. Il existe une littérature abondante, qui montre l'extrême variété des effets du NGF sur la différenciation terminale des neurones sensibles. Outre la poussée axonale, on observe un accroissement spectaculaire de l'activité spécifique des enzymes impliquées dans la 


\section{RÉFÉRENCES}

17. Edgar D, Timpl R, Thoenen $H$. The heparin-binding domain of laminin is responsible for its effects on neurite outgrowth and neuronal survival. $E M B O J 1984 ; 3$ : 1463-8.

18. Monard D. Cell-derived proteases and protease inhibitors as regulators of neurite outgrowth. Trends Neurosi $1988 ; 11$ : 541-4.

19. Anderson DJ, Axel R. A bipotential neuroendocrine precursor whose choice of cell fate is determined by NGF and glucocorticoids. Cell 1986; 47 : 1079-90.

20. Rohrer H, Heumann R, Thoenen $H$ The synthesis of nerve growth factor (NGF) in developing skin is independent of innervation. Dev Biol 1988 ; 128 : 240-4.

21. Ayer-Lelièvre C, Olson L, Ebendal T, Seiger A, Persson H. Expression of the beta nerve growth factor gene in hippocampal neurons. Science $1988 ; 240: 1339-41$.

22. Senut MC, Lamour Y, Lee J, Brachet P, Dicou E. Neuronal localization of the nerve growth factor precursor-like immunoreactivity in the rat brain. Int $J$ Dev Neurosci $1990 ; 8$ : 65-80.

23. Wion D, Barrand P, Dicou E, Scott J Brachet $P$. Serum and thyroid hormones T3 and $\mathrm{T} 4$ regulate nerve growth factor mRNA levels in mouse L cells. FEBS Lett 1985 ; 189: 27-41.

24. Houlgatte $R$, Wion $D$, Brachet $P$. Serum contains a macromolecular effector promoting the synthesis of nerve growth factor (NGF) in L cells. Biochem Biophys Res Commun $1988 ; 150$ : 723-30.

25. Houlgatte $R$, Wion $D$, Brachet $P$. Levels of nerve growth factor secreted by rat primary fibroblasts and iris transplants are influenced by serum and glucocorticoids. Dev Brain Res 1989; 47 : 171-9.

26. Lindholm D, Heumann R, Meyer M, Thoenen $H$. Interkeukin I regulates synthesis of nerve growth factor in non-neuronal cells of rat sciatic nerve. Nature $1987 ; 330$ : 658-9.

27. Wion $D$, Houlgatte $R$, Brachet $P$ Phorbol 12 myristate 13-acetate (TPA) increases the expression of the nerve growth factor (NGF) gene in mouse L-929 fibroblasts. FEBS Lett 1990 ; 262 : 42-4.

28. Hengerer $B$, Lindholm D, Heumann $R$, Rüther $U$, Wagner EF, Thoenen $H$. Lesion-induced increase in nerve growth factor mRNA is mediated by c-fos. Proc Natl Acad Sci USA 1990 ; 87 : 3899-903.

29. Houlgatte R, Mallat M, Brachet $P$, Prochiantz A. Secretion of nerve growth factor in cultures of glial cells and neurons derived from different regions of the mouse brain. J Neurosci Res 1989; 24 : 143-52.

30. Gall CM, Isackson PJ. Limbic seizures increase neuronal production of messenger RNA for nerve growth factor. Science 1989 ; 245 : 758-61.

31. Sofroniew MV, Galletly NP, Isacson O, Svendsen CV. Survival of adult basal forebrain cholinergic neurons after loss of target neurons. Science $1990 ; 247$ : 338-42. 32. Hefti F. Nerve growth factor promotes survival of septal cholinergic neurons after fimbrial transection. J. Neurosci $1986 ; 6$ : synthèse de catécholamines dans les neurones sympathiques, ou de neuropeptides, dont la substance $P$ dans les neurones sensoriels. Des traitements avec des anticorps anti-NGF ou l'immunisation d'animaux par le facteur aboutit au résultat inverse. Dans le système nerveux central, le NGF augmente l'activité spécifique de la choline acétyltransférase, chargée de la synthèse d'acétylcholine, dans les neurones cholinergiques du septum, de la bande diagonale de Broca, du noyau de Meynert, ou encore les interneurones cholinergiques du striatum. Faire le catalogue des informations disponibles sur les effets du NGF, qui concernent la plupart des fonctions métaboliques de la cellule nerveuse, sort certainement des objectifs de cet article. Mais on peut en revanche signaler l'existence de mécanismes modulant les effets du NGF.

Certaines molécules, qui n'ont aucun pouvoir particulier sur la survie ou la morphologie des neurones, modifient leur contenu en neurotransmetteurs. On trouve, par exemple, dans des surnageants de culture de cellules musculaires, une glycoprotéine qui accroît les compétences cholinergiques des neurones sympathiques de rat nouveau-né sans qu'elle exerce d'action trophique proprement dite, laquelle reste l'apanage du NGF [15]. Le ciliary neurotrophic factor (CNTF), qui ne provoque pas de pousse neuritique et n'a qu'un effet très limité sur la survie in vitro de ces mêmes neurones, stimule également leurs propriétés cholinergiques, en la présence obligée de NGF pour éviter leur mort [16].

Par ailleurs, des facteurs d'attachement comme la laminine [17] ou des molécules apparentées augmentent les effets du NGF sur la survie neuronale in vitro et facilitent la pousse neuritique. D'autres macromolécules, comme certaines protéases et leurs inhibiteurs, favorisent la progression

Figure 2. Mise en évidence de I'ARNm du NGF par hybridation in situ D (haut) et du précurseur du NGF (proNGF) par immunohistochimie (bas) dans I'hippocampe du cerveau de rat. L'hybridation in situ a été réalisée avec une sonde d'ADNc du NGF [21]. L'immunohistochimie met en jeu des anticorps dirigés contre des peptides de synthèse reproduisant des séquences d'acides aminés du proNGF [22]. Diverses techniques ont démontré que le matériel mis en évidence dans l'un et l'autre cas, était d'origine neuronale. (Documents : C. Ayer-Lelievre; M.C. Senut et E. Dicou.) des cônes de croissance et, par conséquent, la réponse morphologique [18]. Si le NGF est apparemment dépourvu d'effet sur les cellules de la crête neurale, son action sur les neuroblastes primitifs peut être modulée, notamment par les glucocorticoïdes. En présence de NGF, les neuroblastes adrénergiques se différencient en neurones sympathiques. Mais une exposition conjointe aux glucocorticoïdes, qui se produit dans la glande surrénale, aboutit à la genèse des cellules chromaffines qui se trouvent dans la partie médullaire de cet organe [19]. Et in vitro, on peut faire pousser des neurites à ces dernières cellules en présence de NGF, ou inhiber l'effet du facteur sur l'excroissance neuritique des neurones sympathiques en ajoutant des glucocorticoïdes au milieu des cultures. Signalons enfin que chez certains neuroblastomes, l'apparition des récepteurs à haute affinité du NGF est induite par l'insulin-like growth factor II (IGF II). Par voie de conséquence, IGF II et NGF exercent des effets synergiques.

\section{Cellule nerveuse et tissu cible : un dialogue de sourds?}

On s'attendait, dans un contexte aussi subtil, à ce qu'existât un dialogue direct entre tissu et neurone. Or cela ne paraît pas être le cas. La résection des afférences sympathiques innervant l'iris ou le cœur ne provoque aucun changement des teneurs en ARNm NGF dans ces tissus. Dans le cerveau, la section de la voie septo-hippocampale au niveau de la accroissement faible (et contesté) des teneurs en messager NGF dans l'hippocampe. Les contenus tissulaires en protéine NGF augmentent tant dans l'iris, le cœur que dans l'hippocampe, mais ce phénomène peut s'expliquer par la destruction du fimbria-fornix ne provoque qu'un 


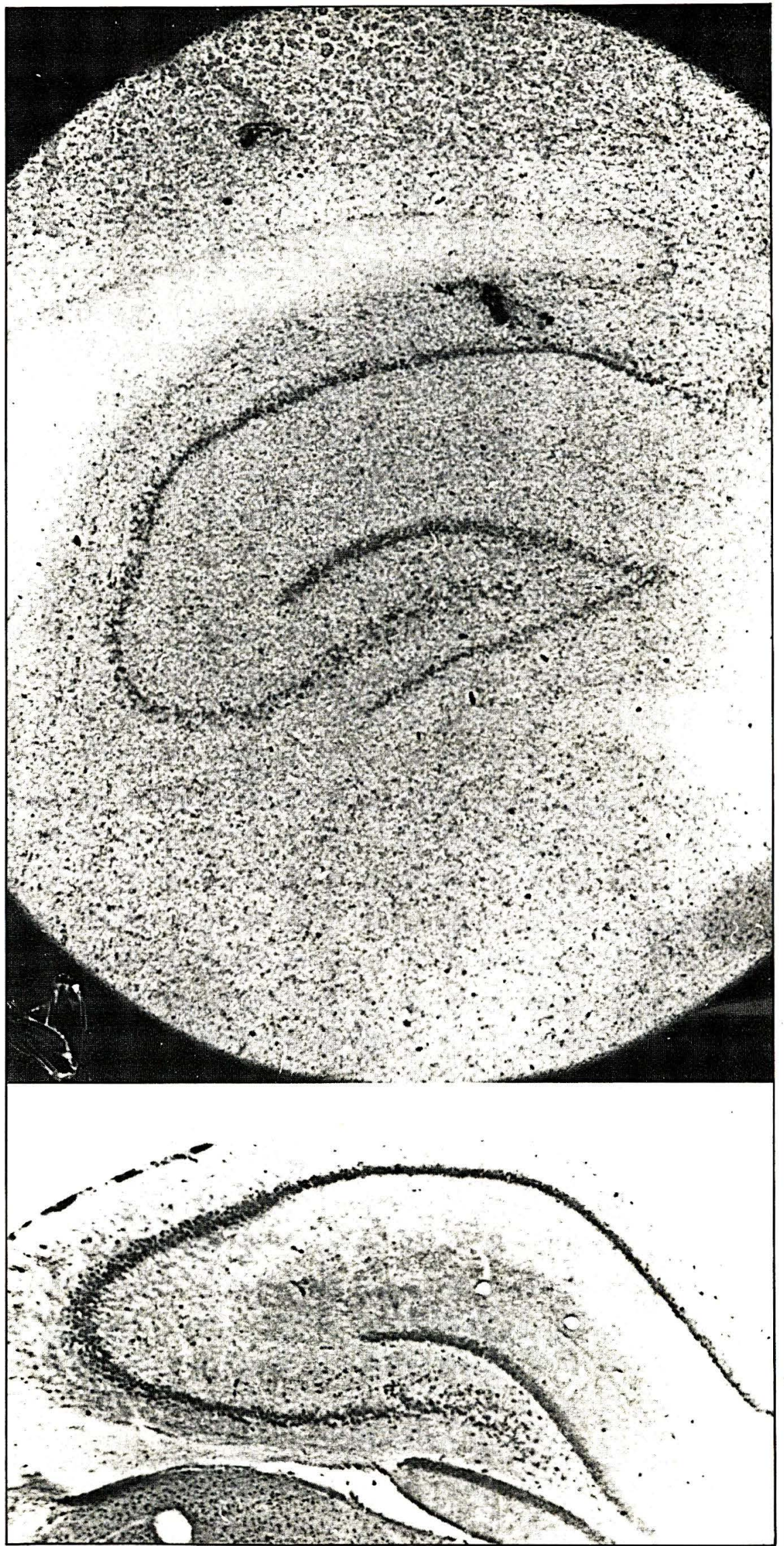

système de capture et de transport axonal rétrograde du NGF par des terminaisons nerveuses en dégénérescence. Des observations parallèles sont faites au cours du développement. La synthèse de NGF par un tissu périphérique, comme la lèvre supportant la moustache du rongeur, est enclenchée au moment où ce tissu est envahi par une innervation sensorielle abondante. Or, l'élimination préalable des neurones assurant l'innervation de la peau ne modifie en rien l'intensité de la vague de synthèse ou le moment où elle prend place [20]. Tout se passe comme si la cellule nerveuse était incapable de transmettre à ses cibles une information sur sa présence et son état métabolique ; tandis que la capacité d'un tissu à produire du NGF relève d'un programme indépendant de la présence ou de l'absence d'une innervation appropriée.

Pourtant, le dialogue existe, mais il met en jeu des intermédiaires.

\section{Lieux de synthèse et apercu sur sa régulation}

Dans les tissus périphériques, le NGF est produit par des cellules non neuronales ; en fait, on ignore toujours lesquelles, étant donné le faible niveau de synthèse. Dans le cerveau, les hybridations in situ [21] ou l'immunohistologie du précurseur du NGF [22] indiquent que les cellules productrices sont des neurones (figure 2). La glie ne paraît pas en produire, du moins en quantité décelable par ces techniques, et dans un cerveau normal. Tout change de façon surprenante dès que l'on passe in vitro, où de très nombreux types cellulaires sont susceptibles de produire du NGF : fibroblastes, cellules de Schwann, myoblastes, adipocytes, astrocytes... De même, la synthèse dans un organe transplanté in vitro peut être stimulée près de 100 fois. Enfin, une stimulation de synthèse du même ordre de grandeur est observée suite à des processus lésionnels in vivo, par exemple au niveau de la portion distale du nerf sciatique sectionné [12]. Un examen attentif des situations existantes in vitro, où la composition du milieu peut être strictement contrôlée, nous 


\section{RÉFÉRENCES}

33. Williams L, Varon S, Peterson S. Continuous infusion of nerve growth factor prevent basal forebrain neuronal death after fimbria-fornix transection. Proc Natl Acad Si USA 1986 ; 83 : 9231-5.

34. Coyle TJ, Price D, De Long MR. Alzheimer's disease: a disorder of cortical cholinergic innervation. Science 1983; 219 : 1184-90.

35. Goedert M, Fine A, Hunt SP, Ulrich A. Nerve growth factor mRNA in peripheral and central tissues and in the human central nervous system: lesion effects in the rat brain and levels in Alzheimer's disease. Mol Brain Res 1986 ; 1 : 85-92.

36. Goedert M, Fine A, Dawbarn D, Wilcock GK, Chao MV. Nerve growth factor receptor mRNA distribution in human brain: normal levels in basal forebrain in Alzheimer's disease. Mol Brain Res 1989 ; 5: $1-7$.

37. Fischer $\mathbf{W}$, Wictorin $\mathrm{K}$, Björklund $\mathrm{A}$, Williams LR, Varon S, Gage FH. Amelioration of cholinergic neuron atrophy and spatial memory impairment in aged rats by nerve growth factor. Nature 1987 ; 329 : 65-9.

38. Chakrabarti S, Sima AAF, Lee J, Brachet $P$, Dicou E. Nerve growth factor (NGF), proNGF and NGF receptor-like immunoreactivity in BB-rat retina. Brain Res $1990 ; 523: 11-5$.

39. Levi-Montalcini R, Aloe L, Mugnaini E, Oesch F, Thoenen $H$. Nerve growth factor induces volume increase and enhances tyrosine hydroxylase synthesis in chemically axotomized sympathetic ganglia of newborn rats. Proc Natl Acad Sai USA 1975; 72 : 595-9.

40. Johnson Jr EM. Destruction of the sympathetic nervous system in neonatal rats and hamsters by vinblastine: prevention by concomitant administration of nerve growth factor. Brain Res 1978 ; 141 : 105-18.

41. Aloe L. Intraocular herpes simplex virus injection in neonatal rats induces sympathetic nerve cell destruction: effect of nerve growth factor. Int $J$ Dev Neurosci 1987 ; 5 : 357-66.

42. Rosenberg ME, Friedman T, Robertson $\mathrm{RC}$, et al. Grafting genetically modified cells to damaged brain: restorative effects of NGF expression. Science 1988 ; 242 : 1575-8. 43. Ernfors P, Ebendal T, Olson L, Mouton $\mathrm{P}$, Strömberg I, Persson $\mathrm{H}$. A cell line producing recombinant nerve growth factor evokes growth responses in intrinsic and grafted central cholinergic neurons. Proc Natl Acad Sci USA 1989 ; 86 : 4756-60.

44. Hohn A, Leibrock J, Bailey K, Barde YA. Identification and characterization of a novel member of the nerve growth factor/brain-derived neurothrophic factor family. Nature 1990 ; 344 : 339-41. a révélé que le sérum est responsable de l'accroissement de la synthèse de NGF [23] (figure 3, p. 861). Il contient au moins deux effecteurs susceptibles d'accroître la production de ce facteur dans des fibroblastes, des astrocytes ou des transplants d'organes [24, 25]. Ces effecteurs sériques pourraient agir rapidement in vivo à la suite de la rupture des capillaires sanguins, par exemple après la transection d'un nerf, ou à l'occasion d'une situation traumatique dans un organe quelconque. Par ailleurs, l'interleukine I, sécrétée par des macrophages qui envahissent les portions d'un nerf en cours de dégénérescence wallérienne, est également capable de stimuler la synthèse de NGF par les cellules de Schwann ou des fibroblastes présents dans la gaine [26]. Cette lymphokine peut, par conséquent, prendre le relais d'éléments sériques [25]. L'action du sérum sur le niveau d'expression du gène NGF met notamment en jeu une protéine kinase $\mathrm{C}$ et l'activation de proto-oncogènes. Elle est en revanche contrecarrée par des agents anti-inflammatoires, dont la dexaméthasone et la cortisone, qui abaissent les niveaux de l'ARNm du NGF dans des conditions où le sérum l'augmente [25, 27]. Ce sont notamment les proto-oncogènes fos et jun qui sont impliqués dans l'accroissement de la synthèse NGF tant in vitro qu'in vivo, après transection du nerf sciatique [28].

In vitro, les astrocytes prélevés à partir de diverses structures du cerveau de souriceau nouveau-né sécrètent des quantités identiques de NGF. A l'instar des nombreux types de cellules périphériques examinés, cet effet n'est obtenu qu'en présence de sérum. Les neurones, quant à eux, ont un comportement différent, puisqu'ils se distinguent in vitro par leur capacité à libérer du facteur : des neurones embryonnaires striataux sécrètent, par exemple, beaucoup plus de NGF que des neurones corticaux et ce, dans un milieu dépourvu de sérum [29]. La réponse des astrocytes cultivés en présence de sérum suggère que ces cellules pourraient devenir productrices de NGF in vivo après traumatisme. De fait, l'ischémie ou certaines lésions chirurgicales aboutissent à des augmenta- tions des teneurs en NGF, qui pourraient provenir de la glie. Les informations restent encore très parcellaires sur ce sujet : une lésion, induite par un choc électrolytique au niveau du girus denté, provoque apparemment une augmentation spectaculaire (près de 20 fois) de l'ARNm NGF au niveau de l'hippocampe [30]. Les cellules responsables de cet effet restent à caractériser.

\section{Survie neuronale et implication en pathologie neurodégénérative}

Chez l'animal adulte, la destruction des neurones producteurs de NGF dans l'hippocampe, grâce à des injections in situ de l'acide aminé excitateur N-méthyl-D-aspartate, n'entraîne pas une mortalité particulièrement élevée des neurones cholinergiques du septum [31]. Certes, on ignore si du NGF n'est pas fourni par une autre source dans ces conditions (cellules gliales, par exemple). Ce résultat suggère néanmoins que la maintenance de l'innervation cholinergique issue du septum n'est pas strictement dépendante de la présence de NGF, à l'instar de l'innervation périphérique sympathique ou sensorielle de l'animal adulte.

En revanche, il est frappant de constater que les neurones cholinergiques septaux meurent après axotomie au niveau de la fimbria-fornix, cette structure qui sépare les corps cellulaires de leurs terminaisons situées dans l'hippocampe. Cet effet létal est en large partie empêché si du NGF est perfusé de façon intraventriculaire $[32,33]$. C'est donc à nouveau lors d'un processus lésionnel affectant directement la cellule nerveuse que, chez l'adulte, la présence de NGF revêt une importance cruciale pour sa survie. On sait que la maladie d'Alzheimer se caractérise notamment par un déficit d'acétylcholine et une mort des neurones cholinergiques des régions basales du cerveau antérieur [34]. Les niveaux de l'ARNm du NGF ou de son récepteur (basse affinité) ne paraissent pas sensiblement différents dans des cerveaux normaux ou pathologiques $[35,36]$. On ignore encore si la maturation du proNGF ou si la synthèse et le fonctionnement 

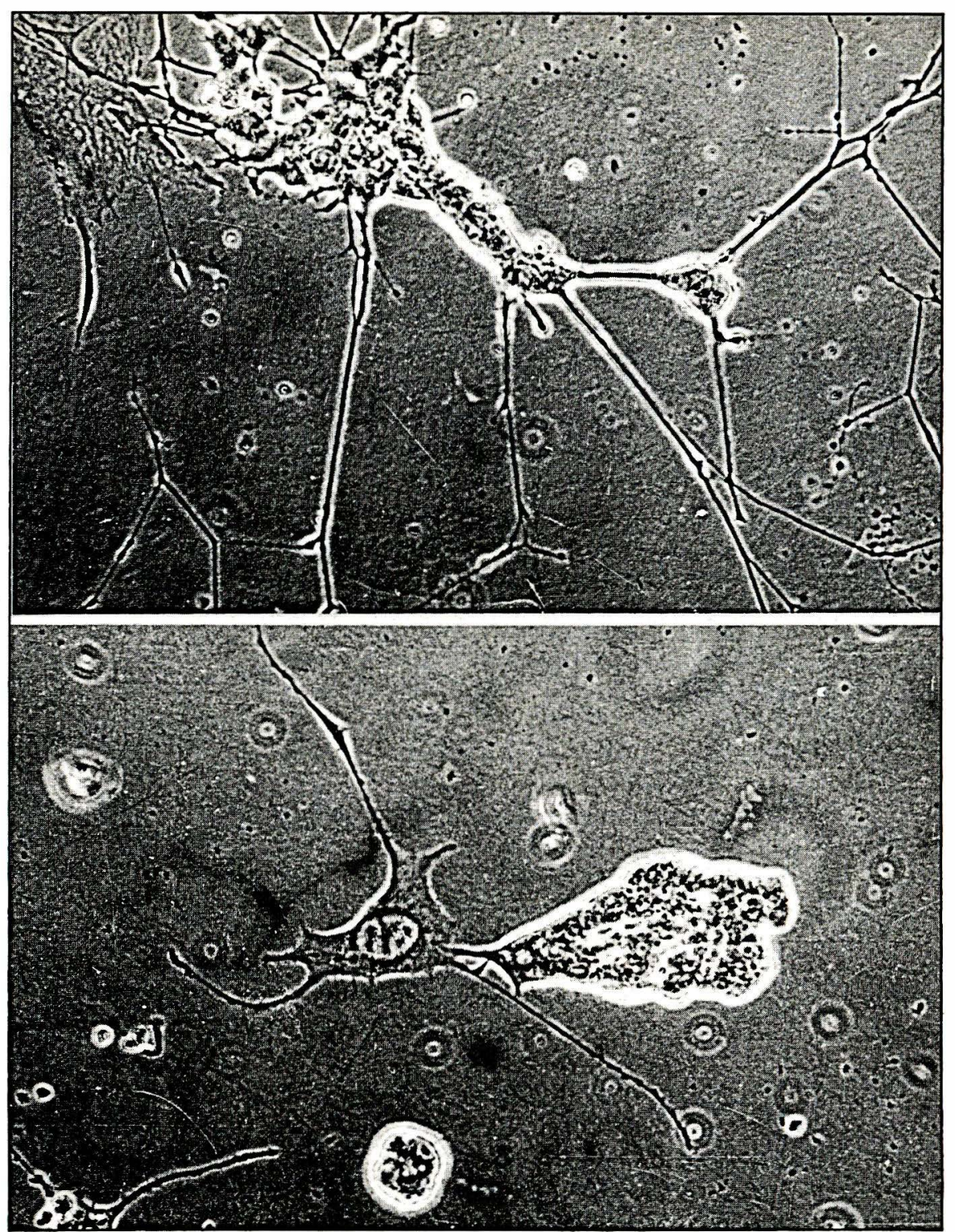

Figure 3. Visualisation de l'effet du sérum sur la synthèse de NGF. Des co-cultures mettent en jeu des fibroblastes producteurs de NGF lles cellules L-929, dont la prolifération a été bloquée par un traitement à la mitomycine) et des cellules d'un phéochromocytome murin, PC12, qui émettent des neurites en présence de NGF. En haut, co-culture réalisée en présence de sérum. Des neurites sont émises par un agrégat de cellules PC12 situé juste à côté d'un fibroblaste visible dans l'angle supérieur gauche. En bas, les cellules sont maintenues dans un milieu défini, où elles restent parfaitement viables. Aucune neurite n'est observée en dépit de la proximité de l'agrégat de cellules PC12 et d'un fibroblaste, comme dans le cliché du haut. L'induction de la synthèse de NGF par le sérum est vérifiable au niveau moléculaire par un dosage direct de la protéine par méthode ELISA, ou de son ARNm par hybridation avec un ADNc NGF [23]. (Document : P. Brachet et E. Dicou.) du récepteur à haute affinité sont normaux. Mais il est cependant peu tentant d'attribuer la mort des neurones cholinergiques à un problème lié à la production ou au mode d'action du NGF. Il reste qu'un apport accru en NGF pourrait avoir un effet bénéfique chez les patients affligés de démence sénile de type Alzheimer. Le fait que la perfusion de NGF à des rats âgés inverse une relative atrophie des neurones cholinergiques du cerveau basal antérieur, et aille jusqu'à améliorer les capacités de mémoire de ces animaux [37], abonde sérieusement dans le sens de cette possibilité. Un raisonnement identique peut s'appliquer à d'autres affections, comme la complication neurologique du diabète juvénile insulino-dépendant. Chez l'animal diabétique, l'apport en NGF, tel qu'il a été étudié au niveau de la rétine, ne paraît pas anormal [38] ; mais certains aspects de cette polyneuropathie pourraient éventuellement être atténués grâce à une modulation appropriée de l'apport neurotrophique.

Par ailleurs, on sait déjà que le rôle protecteur du NGF s'exerce dans d'autres situations : son activité trophique est susceptible de contrecarrer l'action toxique de la 6-hydroxydopamine, un poison de l'innervation sympathique, ou de la vinblastine $[39,40]$. Un traitement par du NGF atténue encore les lésions occasionnées au ganglion supérieur cervical sympathique du rat nouveau-né par le virus herpes simplex injecté dans la chambre antérieure de l'œil [41]. L'administration de NGF humain, synthétisé par les voies du génie génétique, est actuellement projetée chez des malades atteints de démence sénile de type Alzheimer. L'intérêt est majeur, mais le protocole se heurte à des difficultés techniques, notamment par le fait que le NGF ne franchit pas la barrière hémato-méningée et qu'il doit être instillé localement dans le cerveau par une pompe microosmotique. En outre, le NGF recombinant a toutes les chances d'être fort cher. Aussi des solutions alternatives sont-elles souhaitables.

Trois voies générales sont étudiées. La première est axée sur la recherche d'agonistes, peptidiques ou non. Une autre possibilité consiste à 
moduler la synthèse de NGF in vitro par des agents pharmacologiques, susceptibles d'accroître ou de diminuer spécifiquement la production de facteur neurotrophique. C'est dans ce contexte qu'il faut situer les efforts accomplis pour caractériser à un niveau moléculaire les mécanismes régulateurs contrôlant l'expression du gène NGF, qui ont été évoqués plus haut. La troisième voie met en jeu les techniques de greffe. Divers rapports récents ont montré que la greffe de cellules productrices de NGF, comme des fibroblastes préalablement programmés par les méthodes du génie génétique, peuvent aboutir à la survie des neurones cholinergiques septaux après transection de la fimbria-fornix, comme à l'accroissement de leur contenu en choline acétyltransférase [42, 43].

L'amélioration des vecteurs d'expression, la transformation de cellules plus appropriées que des fibroblastes (cellules gliales notamment) ou l'obtention d'animaux surproduisant le NGF par les méthodes de la transgénie, constitueront autant de sources de matériel expérimental greffable, d'un grand intérêt potentiel

\section{Summary}

Nerve growth factor (NGF) : involvement in plasticity and functional maintenance of nerve cells

Nerve growth factor (NGF) is a neurotrophic protein which plays a key role in the survival, terminal differentiation and functional maintenance of peripheral sympathetic and sensory neurons. NGF is also produced in the central nervous system where it exerts trophic effects on the cholinergic neurons of the basal forebrain and most probably on other neuronal populations. In peripheral tissues, NGF is synthesized by nonneuronal cells, while in the brain the factor is produced by neuronal cells. These include neurons of the cortex and hippocampus which are targets of the NGF-responsive cholinergic neurons of the basal forebrain. NGF synthesis may be stimulated under different experimental conditions. In vitro, serum or interleukin I are known to promote NGF synthesis while glucocorticoids exert an opposite effect. These regulatory mechanisms are probably part of wound responses which may occur in vivo following various types of lesions, and which participate in regenerative phenomena. In adult animals, NGF remains required for the survival of axotomized peripheral or central neurons. This suggests that promotion of NGF synthesis or administration of exogenous NGF may be helpful in some pathological disorders involving neurodegenerative processes, such as Alzheimer's disease or the diabetes-associated peripheral polyneuropathy. 